\title{
Residence Time, Water Contact, and Age-driven Schistosoma mansoni Infection in Hotspot Communities in Uganda
}

\author{
Arinaitwe Moses, ${ }^{1,2 *}$ Moses Adriko, ${ }^{1}$ Brian Kibwika, ${ }^{2}$ Edridah M. Tukahebwa, ${ }^{1}$ Christina L. Faust, ${ }^{3} \dagger$ and \\ Poppy H. L. Lamberton ${ }^{3} \dagger$ \\ ${ }^{1}$ Vector Borne and Neglected Tropical Diseases Control Division, Ministry of Health, Kampala, Uganda; ${ }^{2}$ Cavendish University Uganda, Kampala, \\ Uganda; ${ }^{3}$ Institute for Biodiversity, Animal Health and Comparative Medicine and Wellcome Centre for Integrative Parasitology, University of \\ Glasgow, Glasgow, United Kingdom
}

\begin{abstract}
Schistosomiasis is the second most important parasitic infection after malaria in terms of its socioeconomic impact and is endemic in 78 countries. It affects more than 240 million people worldwide, with $90 \%$ of cases occurring in sub-Saharan Africa. In Uganda, Schistosoma mansoni is the most common species, with more than seven million people infected and 17 million living at risk despite mass drug administration (MDA) of praziquantel initiated more than 16 years ago. There has been a shift in the WHO schistosomiasis goals from controlling morbidity to elimination as a public health problem. Understanding the drivers of infection in persistent transmission hotspots despite ongoing control interventions is paramount. We conducted a cross-sectional epidemiological study of 381 individuals in Bugoto community, Mayuge district, Eastern Uganda, along with a structured survey to ascertain drivers of $S$. mansoni infection. Bugoto has had community-wide MDA since 2004. We detected a S. mansoni prevalence of $52 \%$ across the whole community and a prevalence of $71 \%$ in school-age children. This qualifies Bugoto as a highly endemic community according to WHO guidelines. Using a multivariate logistic regression, we found that $S$. mansoni infection was best explained by age group, longer residence times, and any daily contact with lake water. Schistosoma mansoni infection remains a large burden across this community. This study identifies opportunities for interventions that reduce lake water contact, expand treatment eligibility to all at risk, and improve MDA coverage for long-term residents in these settings to control schistosomiasis in persistent transmission hotspots.
\end{abstract}

\section{INTRODUCTION}

Schistosomiasis, which is the second most important parasitic infection after malaria in terms of its socioeconomic impact, is responsible for the loss of an estimated 4.5 million disability-adjusted life years (DALYs) worldwide. ${ }^{1}$ Schistosomiasis, including both intestinal and urinary forms of the disease, occurs in 78 countries across the globe. An estimated 240 million people are infected, with more than 779 million living at risk globally. ${ }^{2}$ The majority of those infected and those at risk for infection live in low-income countries, ${ }^{3}$ and approximately $80 \%$ of the morbidity occurs in impoverished communities and households in sub-Saharan Africa. Within Uganda, 91 of the 134 districts are endemic for intestinal schistosomiasis caused by Schistosoma mansoni, and the eastern region, especially along Lake Victoria, has one of the highest S. mansoni burdens worldwide. ${ }^{4-7}$ Schistosoma haematobium is only endemic in the five districts of the Lango region in northern Uganda. ${ }^{8}$

Schistosomiasis is considered a highly focal disease. ${ }^{9}$ High endemicity communities, defined by the WHO as having a prevalence of more than $50 \%$ in school-age children (SAC), can be found immediately adjacent to communities with low endemicity ( $<10 \%$ SAC). ${ }^{10}$ This focality is partly attributable to the presence of freshwater environments, suitable intermediate host snails, and inadequate sanitation. Schistosoma mansoni ova in infected human feces hatch into miracidia when in contact with freshwater. Miracidia undergo asexual reproduction and several developmental stages in Biomphalaria snails; they are shed into the water

*Address correspondence to Arinaitwe Moses, Vector Borne and Neglected Tropical Diseases Control Division, Ministry of Health, P.O. Box 1661, Kampala, Uganda. E-mail: moses0772359814@ gmail.com

†These authors contributed equally to this work. as an infective-stage cercariae. ${ }^{8}$ Humans become infected with S. mansoni when they contact water harboring these free-swimming cercariae.

Research has investigated both individual and community risk factors that influence schistosomiasis infection intensity and prevalence. Ecological features, including snail habitat and water conditions, are correlated with the presence and prevalence of schistosomiasis at the community level. ${ }^{11}$ Behavioral factors have been shown to influence individual risk, particularly swimming, bathing, and washing clothes in open water sources. ${ }^{12,13}$ Hygiene behaviors, such as open defecation, can lead to high community levels of infection with the disease, although this behavior is not directly linked to the individual's risk for infection. ${ }^{14}$ Certain occupational activities, such as fishing and agricultural practices that involve irrigation and rice paddy growing, increase risk for infection. ${ }^{15}$ In addition, socioeconomic levels at both the family level and individual level influence schistosomiasis infection risk, as reported by Muhumuza et al. ${ }^{16}$ in Uganda.

Within Uganda, the national Bilharzia and Worm Control Program aimed to control schistosomiasis with the goal of elimination of the disease as a public health problem by 2020. ${ }^{17}$ However, as in most other countries, this was not accomplished. The WHO 2021-2030 neglected tropical disease roadmap now aims for $100 \%$ of endemic countries to have reached this goal by $2030 .^{20}$ Mass drug administration (MDA) of praziquantel, health education, access to clean and safe water, and sanitation improvements are strategies recommended by the $\mathrm{WHO}$ to control the disease. As in many African countries, MDA is the mainstay of control in Uganda. This strategy uses WHO guidelines for treatment coverage and aims for $75 \%$ coverage in highly endemic communities (where the prevalence in SAC is $>50 \%$ ) for all members 5 years of age and older. ${ }^{18}$ Although the control program has successfully reduced the burden of the disease, particularly when it was first intitiated, ${ }^{19}$ reduced 
efficacy of praziquantel, ${ }^{6}$ transmission bouncebacks, ${ }^{20}$ high reinfection rates, ${ }^{21}$ and low treatment coverage ${ }^{25}$ are real threats to the continued progress in Uganda and elsewhere. According to cluster randomized trials performed in other countries through a program called SCORE (Schistosomiasis Consortium for Operational Research and Evaluation), particular villages have had persistent high intensity and/or prevalence despite repeated MDA and are referred to as persistent hotspots. ${ }^{22}$ Bugoto community, like many others in Uganda, where our study was performed, has maintained high prevalence despite more than 14 years of MDA and remains a highly endemic community according to WHO endemicity categorization.

With the recent shift in focus by the WHO from morbidity control to elimination as a public health problem, ${ }^{23}$ there is a renewed need to understand drivers of disease transmission in communities that are not responding to standard control interventions. Understanding such drivers would aid disease control through the reorientation of interventions, the development of novel individual-focused and community-focused interventions, and informing program managers of alternative options for control. Schistosomiasis infection can be acquired in environments where infectious vectors are found; therefore, the infection risk is dependent on not only individual factors but also contributions to onward transmission by other members in the community at large. ${ }^{24}$ Lack of access to adequate sanitation facilities can lead to individuals defecating in the environment, thus increasing the risk and transmission of schistosomiasis to others. The main objectives of this study were to improve our understanding of the drivers of infection among individuals as well as quantifying risk factors in a persistent transmission hotspot in Eastern Uganda that has undergone praziquantel MDA since 2003.

\section{METHODS AND PROCEDURES}

Study area. The study was conducted in Bugoto community, which consists of two villages, Bugoto $A$ and Bugoto $B$, on the shores of Lake Victoria in Bukabooli sub-county, Mayuge District, Eastern Uganda. Bugoto community is served by a level-two government health center, one government primary school, and a few smaller private primary schools. A household census conducted in February 2017 in Bugoto A and Bugoto B demonstrated that these are predominately small-scale agriculture and fishing communities, respectively. ${ }^{25}$ These villages are representative of small rural communities along water bodies. ${ }^{26}$ National school-based MDA of praziquantel began in 2003; in 2004, it was scaled-up to cover the whole community on an annual basis. In 2019, 1 year after data collection for this study, community-wide MDA was scaled-up again to biannual treatment. Bugoto community was chosen for this study because it remains highly endemic for intestinal schistosomiasis $^{6,7,27}$ and had undergone annual community-wide MDA of praziquantel for more than 14 years at the time when the study was undertaken, which is the maximum time for any community in Uganda. Despite receiving community-wide MDA, transmission and infection remain high; therefore, additional interventions are needed in this area and in similar areas.

Survey design and data collection. The disease burden across Bugoto community was estimated at $50 \%$ according to previous studies. Using a sampling error of $0.5 \%$ at a $95 \% \mathrm{Cl}$ and $z$-score of $1.96,{ }^{28}$ the minimum sample size required to differentiate the infection prevalence and lead to understanding the drivers of acquiring schistosomiasis infection within this community was calculated as 384 individuals. All individual members of the community were first registered during a community-wide household survey in February 2017.25 Epidemiological cohorts were recruited in March 2017 (SAC 6-14 years of age) and November 2017 (preschool-age children [PSAC] 9 months-5 years and individuals older than 14 years) (Figure 1). Individuals were randomly selected from the community register to represent equal sex and age ratios. The Uganda population and housing report ${ }^{26}$ offers estimates of the age structure in 5-year increments (approximating each age group); according to that report, $19.5 \%$ are PSAC (defined as younger than 5 years; however, PSAC in Uganda are commonly younger than 6 years), 34\% are SAC (age 5-14.9 years), and $46.3 \%$ are "adults" who are 15 years or older. Individuals were proportionally sampled from these three age groups but defined as slightly different age categories (Figure 1) as follows: 75 PSAC, 131 SAC, and 178 adults. Children younger than 9 months were not sampled because of ethical constraints associated with treatment. Follow-up of the participants to obtain behavioral and knowledge data was conducted in March 2018. The subset for these behavioral and knowledge surveys were randomly selected from the individuals who had previously completed the household survey and provided samples for the epidemiological crosssectional survey in March 2017 for SAC and in November 2017 for PSAC and adults (Figure 1).

At the time of the initial recruitment, and at the time of each subsequent follow-up survey, study procedures were explained to the participants; full ethical consent and assent were required for participation. Children younger than 15 years were consented by their parents/guardians, and children 8 years and older also provided assent in addition to parental/guardian consent. A semi-structured questionnaire was administered by Vector Control Officers to all consenting individuals. Parents, guardians, and/or older siblings completed questionnaires on behalf of children younger than 5 years. Questions were designed to determine specific individual demographic, behavioral, environmental, and socioeconomic characteristics related to the acquisition of intestinal schistosomiasis (Table 1). Demographic variables collected included age, sex, and religion. Environmental variables included the number of years an individual has been a resident of the community and the approximate distance of the house from the lake shoreline.

Contracting schistosomiasis involves contact with water with intermediate vector snails that are shedding larval cercariae; however, not all water contact sites have an equal risk. Mapping out frequently visited contact sites along the lake shoreline for this community and differentiating risk (through infection status) would help identify transmission sites where control interventions could be targeted. Based on 15 years of working in this community, we divided water contact sites by the types of activities conducted there. These were also partitioned into the following different ecological zones: zone 1, swampy shoreline where some community members go to get water as well as grow rice; zone 2 , rocky far end of the community extending into lake 


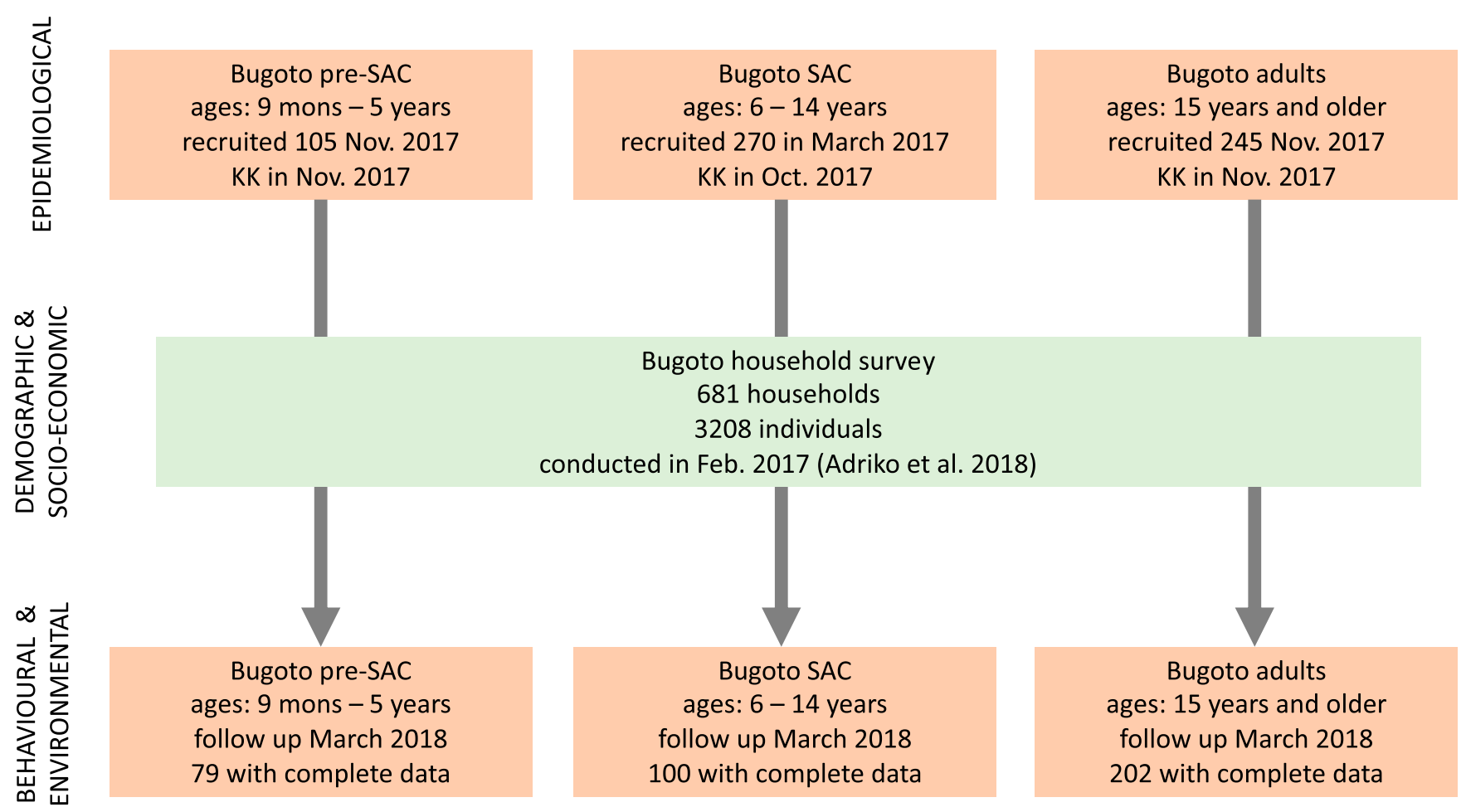

FlgURE 1. Recruitment and data collection flow. Timing and sample size of recruited individuals during each data collection event. This figure appears in color at www.ajtmh.org.

and $10 \mathrm{~m}$ from the shoreline that is used by community members for fishing and transit to islands; and zone 3, open lake shoreline stretching more than $300 \mathrm{~m}$ but not exceeding $10 \mathrm{~m}$ from the shoreline, with some floating vegetation, that is used mostly for swimming, gathering household water, and transit (Supplemental Figure S1).

The reported behavioral variables in this survey focused on lake water contact and MDA compliance. Reported lake water contact (frequency, duration, and activities) was

TABLE 1

Variables collected during the survey

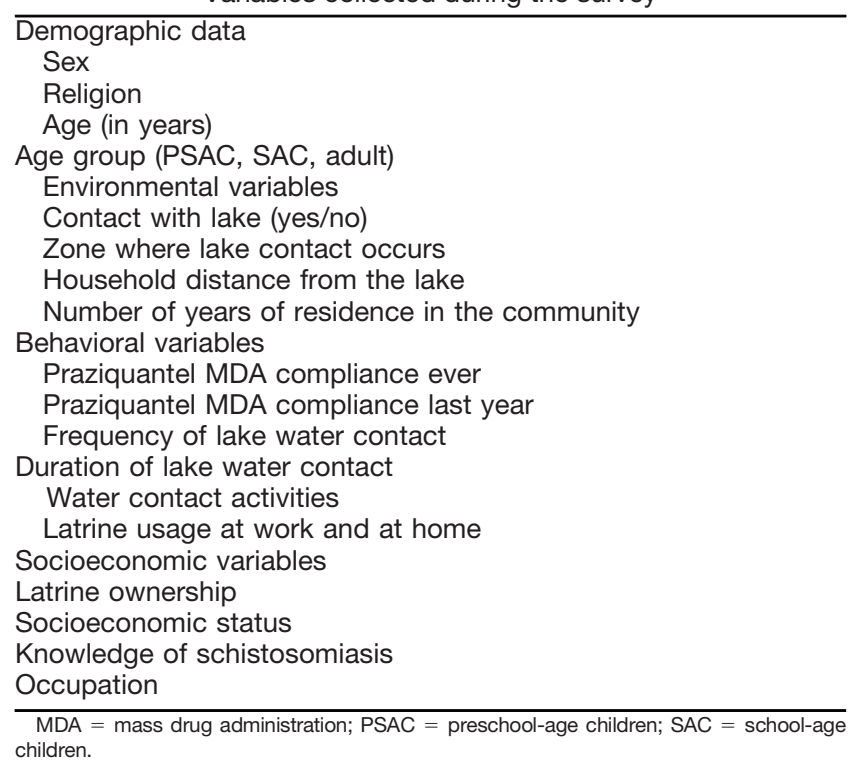

recorded. Praziquantel MDA compliance was assessed based on individual recall both within the previous MDA campaign (July 2017) and ever during their lifetime. Survey questions also collected data regarding latrine usage at home and work.

Intestinal schistosomiasis is a neglected tropical disease that is strongly associated with low socioeconomic development. To ascertain the socioeconomic status of the household, we used an established method to assign values to different construction material within households ${ }^{29}$ (house floors: mud $=1$, cement $=2$, tiles $=3$; walls: mud $=1$, bricks $=2$, bricks and plaster $=3$; and roofing: grass-thatched $=1$; papyrus $=2$; iron sheets $=3$ ). In addition, we collected information regarding latrine ownership, water source, and level of education. We divided latrine ownership into three categories: no latrine $=0$; shared latrine $=2$; or private latrine $=3$. We scored water sources as follows: lake $=1$; borehole $=2$; and piped water $=$ 3. The level of education was scored as follows: no education $=0$; primary level $=2$; and secondary level or more $=3$. Then, we used these scores to create the socioeconomic status of the household (household head and family members) based on the work by Filmer and Pritchett. ${ }^{30}$

Finally, we asked participants questions about modes of transmission, effects of infection on individuals, and types of control methods to ascertain awareness and knowledge of schistosomiasis (see Supplemental Information for full questionnaires).

Epidemiological status. We asked each participant to provide stool samples in October 2017 (SAC) and November 2017 (PSAC and adults). We processed samples using the Kato-Katz thick smear technique. ${ }^{28}$ To improve the accuracy of infection status for low-intensity infections, three 
consecutive daily stool samples were collected from each participant. These samples were prepared on duplicate slides and read by highly trained Vector Control Officers from the Ministry of Health under a compound microscope ( $\times 10$ objective). Before being approved to read slides, Vector Control Officers must complete a course that allows them to learn how to evaluate the sensitivity and specificity of Kato-Katz parasitological readings. We used to recheck $25 \%$ of slides, but errors were rare and did not significantly affect the prevalence or intensity measures; therefore, we halted this practice to conserve resources. We did not conduct parasitological surveys of Schistosoma haematobium because it is not endemic in this community or district, despite being repeatedly tested for over the years, including by the authors. We defined a positive schistosomiasis status as the presence of at least one S. mansoni ova on any KatoKatz slide.

Data analysis. We double-entered parasitological and survey data into Excel and cleaned the data. We created univariate logistic regressions to evaluate which demographic, environmental, behavioral, and socioeconomic variables were predictive variables of the infection status. Then, we performed multivariate logistic regression with all significant univariate variables to determine the most important factors influencing the risk of acquiring intestinal schistosomiasis, as measured by presence of eggs in stool. Model selection was performed in a stepwise manner, and the best-fit model was selected using the Akaike information criterion.

Ethical consideration. We conducted this study within the framework of the ongoing Bilharzia and Worm Control Program of Ministry of Health and the European Research Council funded SCHISTO_PERSIST 688088 project (to PHLL). Approvals were obtained from the Vector Control Division Research Ethics Committee (VCDREC/062), Uganda National Council for Science and Technology (UNCST-HS 2193), and Veterinary and Life Science Research Ethics Committee of the University of Glasgow College of Medicine (MVLS 200160068). We also obtained permission from Mayuge district local government administration. We obtained signed/thumbprint informed consent from all adults and the parents or guardians of SAC and PSAC. We obtained assent from all SAC. We treated all SAC and adults diagnosed as schistosomiasis-positive with 40 $\mathrm{mg} / \mathrm{kg}$ praziquantel. Positive PSAC (9 months-5 years of age) were treated with $60 \mathrm{mg} / \mathrm{kg}$. Participants could withdraw themselves or their children from the study at any time without it affecting their access to praziquantel treatment during this study or through community MDA.

\section{RESULTS}

After recruitment was performed and informed ethical consent was obtained, a total of 381 individuals participated in the study; of these, $50.1 \%$ were male (Figure 1, Table 2). A higher proportion of males was infected with S. mansoni $(55.5 \%)$, although the difference was not significant (Table 3).

Of the 381 participants, the age structure mirrored the population average, with $20.7 \%$ PSAC (79/381), 26.2\% SAC (100/381), and 53.0\% adults (202/381). The prevalence of $S$. mansoni in SAC was $71.0 \%$, thus confirming Bugoto as a highly endemic community as defined by the WHO.
TABLE 2

Characteristics of study participants and infection status $(N=381)$

\begin{tabular}{lcr}
\hline \multicolumn{1}{c}{ Group } & Enrolled, $\mathrm{n}(\%)$ & S. mansoni-positive, $\mathrm{n}(\%)$ \\
\hline Age group & & \\
9 months-4.9 years & $79(20.7 \%)$ & $31(39.2 \%)$ \\
$5-14.9$ years & $100(26.2 \%)$ & $71(71.0 \%)$ \\
15 years or older & $202(53.0 \%)$ & $96(47.5 \%)$ \\
Sex & $191(50.1 \%)$ & $106(55.5 \%)$ \\
$\quad$ Male & $190(49.9 \%)$ & $92(48.4 \%)$ \\
Female & $170(44.6 \%)$ & $84(49.4 \%)$ \\
Religion & $76(19.9 \%)$ & $48(63.2 \%)$ \\
Islamic & $103(27.0 \%)$ & $49(47.6 \%)$ \\
Catholic & $32(8.4 \%)$ & $17(53.1 \%)$ \\
Protestant &
\end{tabular}

* Other religious faith included Pentecostal and traditional sect.

Furthermore, more than one-third $(39.2 \%)$ of PSAC had detectable S. mansoni egg infection (Table 2). Across the whole community, the overall prevalence was $52.0 \%$.

The majority of participants $(50.6 \%)$ had access to shared latrines, and a slightly lower percentage (47.3\%) had a private latrine, whereas only $1.0 \%$ reported no latrine access. Up to $22.6 \%$ of participants reported that they did not use latrines at all at work, $23.4 \%$ sometimes used them at work, and $3.2 \%$ reported not using latrines at all. A slightly larger proportion of the participants reported using latrines at home, although this difference was not significant.

Individual risk for infection with $\mathbf{S}$. mansoni. Age in years was not a significant predictor of the $S$. mansoni infection status; however, age group (PSAC, SAC, adult) was. Compared with the $S$. mansoni-negative infection status, according to a univariate analysis, SAC were 4.06-times more likely to be infected with S. mansoni than PSAC (odds ratio [OR]: 0.60; 95\% confidence interval [Cl]: 0.38-0.95) or adults (OR: 1.48; 95\% Cl: 0.87-2.56) (Table 3). The majority of study participants $(83.2 \%)$ reportedly visited the lake. Some only visited the lake a few times a month $(3.4 \%)$ whilst others visited it up to three times per day $(28.3 \%)$. PSAC were less likely to visit the lake $(53.2 \%)$ than SAC (95\%) and adults (89.1\%). Most participants reported their main lake water contact activity was gathering water (27.6\%); other activities included swimming and fishing. The duration of water contact was significantly associated with the $S$. mansoni infection status. Individuals who reported never contacting the water had only a third of the chance of being infected compared to those who contacted the water at all, even if only for less than 5 minutes. Of the individuals who reported not visiting the lake, 25\% were infected with S. mansoni; of those $25 \%$, the majority were PSAC (median age, 2.74 years). The distance from an individual's home to the lake was not significant for predicting S. mansoni infection, nor was the zone where individuals contact lake water. Residing in Bugoto community for 5 or more years was positively associated with the S. mansoni infection risk (OR: 2.66; 95\% Cl: 1.57-4.53) compared with residents who had lived there fewer than 5 years (OR: 0.63; 95\% Cl: 0.43-0.91).

We observed a knowledge gap surrounding schistosomiasis disease, transmission, and control among participants. Fewer than half were able to correctly identify modes of transmission (44.6\%), the impact of infections (33.3\%), or control methods (43.8\%). However, knowledge of all three 
TABLE 3

Univariate analysis results

\begin{tabular}{|c|c|c|c|c|c|}
\hline Category & Variables & OR & $\begin{array}{c}\text { Significance } \\
P<0.1 ;{ }^{\star} P<0.05\end{array}$ & $2.5 \% \mathrm{Cl}$ & $97.5 \% \mathrm{Cl}$ \\
\hline \multicolumn{6}{|l|}{ Demographics } \\
\hline \multirow{2}{*}{ Sex } & Female & 0.94 & & 0.71 & 1.25 \\
\hline & Male & 1.33 & & 0.89 & 1.99 \\
\hline \multirow[t]{2}{*}{ Religion } & Christian (intercept) & 1.18 & & 0.90 & 1.54 \\
\hline & Muslim & 0.83 & & 0.55 & 1.25 \\
\hline \multirow[t]{2}{*}{ Age (years) } & (Intercept) & 1.39 & * & 1.01 & 1.93 \\
\hline & Age & 0.99 & * & 0.98 & 1.00 \\
\hline \multirow{3}{*}{$\begin{array}{l}\text { Age group (PSAC, SAC, } \\
\text { adult) }\end{array}$} & PSAC (intercept) & 0.60 & & 0.38 & 0.95 \\
\hline & SAC & 4.06 & * & 2.20 & 7.65 \\
\hline & Adults & 1.48 & & 0.87 & 2.56 \\
\hline \multicolumn{6}{|l|}{ Environmental } \\
\hline \multirow{3}{*}{$\begin{array}{l}\text { Zone where lake contact } \\
\text { occurs }\end{array}$} & Bushy & 1.50 & & 0.25 & 11.39 \\
\hline & $\begin{array}{l}\text { Landing of the health } \\
\text { facility }\end{array}$ & 0.93 & & 0.12 & 5.71 \\
\hline & Rocky open waters & 0.52 & & 0.06 & 3.70 \\
\hline \multirow{2}{*}{$\begin{array}{l}\text { Household distance from } \\
\text { the lake }\end{array}$} & & 0.82 & & 0.57 & 1.17 \\
\hline & Distance from the lake & 1.51 & * & 0.98 & 2.34 \\
\hline \multirow{3}{*}{$\begin{array}{l}\text { Number of years of } \\
\text { residence in the } \\
\text { community (group) }\end{array}$} & $<5$ years & 0.63 & * & 0.43 & 0.91 \\
\hline & $5-9$ years & 2.66 & * & 1.57 & 4.53 \\
\hline & $\geq 10$ years & 1.89 & * & 1.16 & 3.11 \\
\hline \multirow{2}{*}{$\begin{array}{l}\text { Number of years of } \\
\text { residence in the } \\
\text { community (proportion) }\end{array}$} & (Intercept) & 0.50 & * & 0.31 & 0.80 \\
\hline & Residence & 2.97 & * & 1.64 & 5.46 \\
\hline \multirow[t]{2}{*}{ Village } & A & 1.23 & & 0.96 & 1.57 \\
\hline & $\mathrm{B}$ & 0.66 & & 0.43 & 1.03 \\
\hline \multicolumn{6}{|l|}{ Behavior } \\
\hline \multirow{2}{*}{$\begin{array}{l}\text { PZQ MDA compliance } \\
\text { ever }\end{array}$} & (Intercept) & 0.83 & & 0.59 & 1.17 \\
\hline & $P Z Q$ ever & 1.14 & & 0.72 & 1.82 \\
\hline \multirow{2}{*}{$\begin{array}{l}\text { PZQ compliance last } \\
\text { year }\end{array}$} & (Intercept) & 0.82 & & 0.64 & 1.05 \\
\hline & $P Z Q$ last year & 2.17 & * & 1.42 & 3.35 \\
\hline \multirow{2}{*}{$\begin{array}{l}\text { Contact with lake (yes/ } \\
\text { no) }\end{array}$} & (Intercept) & 0.33 & * & 0.18 & 0.57 \\
\hline & Lake visit & 4.04 & * & 2.25 & 7.63 \\
\hline \multirow{6}{*}{$\begin{array}{l}\text { Frequency of lake water } \\
\text { contact }\end{array}$} & None & 1.51 & * & 1.03 & 2.24 \\
\hline & A few times per month & 1.00 & & 0.60 & 1.67 \\
\hline & A few times per week & 0.73 & & 0.35 & 1.52 \\
\hline & Once per day & 0.23 & * & 0.11 & 0.44 \\
\hline & Twice per day & 0.41 & & 0.12 & 1.32 \\
\hline & Three times per day & 0.51 & & 0.17 & 1.48 \\
\hline \multirow{5}{*}{$\begin{array}{l}\text { Duration of lake water } \\
\text { contact }\end{array}$} & None & 0.33 & * & 0.18 & 0.57 \\
\hline & $<5$ & 3.54 & * & 1.68 & 7.69 \\
\hline & $5-15 \min$ & 4.01 & * & 2.15 & 7.83 \\
\hline & $16-30 \mathrm{~min}$ & 4.73 & * & 2.27 & 10.23 \\
\hline & $>30 \min$ & 3.90 & * & 1.45 & 10.88 \\
\hline \multirow[t]{3}{*}{ Latrine usage at work } & Never & 0.91 & & 0.59 & 1.39 \\
\hline & Sometimes & 0.86 & & 0.47 & 1.55 \\
\hline & Always & 1.48 & & 0.89 & 2.45 \\
\hline \multirow[t]{3}{*}{ Latrine usage at home } & Never & 1.2 & & 0.36 & 4.16 \\
\hline & Sometimes & 0.99 & & 0.27 & 3.48 \\
\hline & Always & 0.86 & & 0.24 & 2.92 \\
\hline
\end{tabular}


TABLE 3

Continued

\begin{tabular}{|c|c|c|c|c|c|}
\hline Category & Variables & OR & $\begin{array}{c}\text { Significance } \\
P<0.1 ;{ }^{*} P<0.05\end{array}$ & $2.5 \% \mathrm{Cl}$ & $97.5 \% \mathrm{Cl}$ \\
\hline \multicolumn{6}{|l|}{ Socioeconomic status } \\
\hline \multirow[t]{3}{*}{ Latrine ownership } & None & 1.00 & & 0.12 & 8.33 \\
\hline & Private & 0.98 & & 0.12 & 8.30 \\
\hline & Shared & 1.19 & & 0.14 & 10.09 \\
\hline \multirow[t]{3}{*}{ Socioeconomic status } & Low & 1.01 & & 0.80 & 1.29 \\
\hline & Medium & 1.21 & & 0.77 & 1.90 \\
\hline & High & $2,087,258.00$ & & 0.00 & NA \\
\hline \multirow{4}{*}{$\begin{array}{l}\text { Knowledge of } \\
\text { schistosomiasis }\end{array}$} & None & 0.80 & & 0.60 & 1.07 \\
\hline & Low & 1.76 & & 0.89 & 3.53 \\
\hline & Medium & 1.80 & * & 0.93 & 3.55 \\
\hline & High & 1.78 & * & 1.11 & 2.88 \\
\hline \multirow[t]{6}{*}{ Education } & None & 0.76 & & 0.52 & 1.12 \\
\hline & Started primary school & 1.79 & & 1.11 & 2.91 \\
\hline & Finished primary school & 0.40 & & 0.11 & 1.23 \\
\hline & Started secondary school & 1.55 & & 0.82 & 2.97 \\
\hline & Finished secondary school & $7,549,132.00$ & & 0.00 & NA \\
\hline & Secondary school or more & 0.66 & & 0.03 & 7.05 \\
\hline
\end{tabular}

MDA = mass drug administration; PSAC = preschool-age children; $\mathrm{PZQ}=$ praziquantel; $\mathrm{SAC}=$ school-age children

was associated with a higher risk of infection with $S$. mansoni (OR: 1.78; 95\% Cl: 1.11-2.88).

Praziquantel MDA compliance rates of the community were below the WHO targets: only $36.2 \%$ of eligible individuals reported receiving treatment during the past year. Praziquantel treatment during the past year was positively associated with S. mansoni infection (OR: 2.17; 95\% Cl: 1.42-3.35). Furthermore, $62.5 \%$ of the eligible participants reported receiving praziquantel at least once during their lifetime, although this was not predictive of the current S. mansoni infection.

Although several factors were important predictors of $S$. mansoni infection when examined in isolation, only a few of these were important when controlling for all possible combinations using a multivariate model. The best-fit model indicated that age group, proportion of lifetime residence, and duration of water contact were all significant predictors of S. mansoni infection (reported as adjusted OR [aOR]). This best-fit model indicated a significant interaction between age group and residence time, meaning that the impact on

TABLE 4

Multivariate analysis

\begin{tabular}{lllr}
\hline & Adjusted OR & $2.50 \%$ & $97.50 \%$ \\
\hline PSAC & 0.7 & 0.05 & 1.94 \\
SAC & 0.0008 & 0.00005 & 0.02 \\
Proportion of residence time & 1.52 & 0.62 & 3.62 \\
Duration of water contact & & & \\
$\quad$ < 5 min & 2.65 & 1.12 & 6.41 \\
$\quad$ 5-15 min & 3.2 & 1.58 & 6.8 \\
16-30 min & 3.85 & 1.67 & 9.22 \\
$\quad>30$ min & 2.59 & 0.84 & 8.14 \\
Age group & & & \\
$\quad$ PSAC: Residence proportion & 1.06 & 0.36 & 1.8 \\
$\quad$ SAC: Residence proportion & 6.73 & 226 & 43.53 \\
\hline PSAC = preschool-age children; SAC = school-age children. & &
\end{tabular}

age is dependent on the proportion of the lifetime spent locally (Table 4). SAC who had lived a small proportion $(<10 \%)$ of their lifetime in Bugoto were at low risk for infection (aOR: 1.06; Cl: 0.36-1.8). However, living an entire lifetime in Bugoto was associated with a 6725-times increase in the infection risk (aOR: 6725; 95\% Cl: 226-43525).

It was observed that any duration of lake water contact, regardless of frequency, was associated with an increased S. mansoni infection risk. Although praziquantel MDA compliance was significantly negatively associated with infection risk when analyzed using univariate logistic regression, it was not present in the best-fit model. Additionally, socioeconomic variables and knowledge of schistosomiasis were not included in the final best-fit model of individual risk for acquiring S. mansoni infection because they were no longer significant.

\section{DISCUSSION}

Schistosomiasis is a complex disease, and its infection risk is closely linked with individual and community behaviors, the environment, and control interventions. Our study focused on a persistent transmission hotspot to identify key drivers of infection and quantify risk factors to target the existing control interventions. We demonstrated that S. mansoni remains a public health problem in Bugoto community (71\% prevalence for SAC) despite more than 14 rounds of praziquantel MDA. The community has several high-risk factors, including inadequate sanitation, highfrequency water contact, and low treatment coverage, ${ }^{25}$ resulting in a high infection rate and endemicity. ${ }^{20}$ Despite these many community-wide factors, we found that the S. mansoni infection risk was best predicted by age group, duration of lake water contact, and residence time within the community, thereby suggesting characteristics to select for 
when designing and targeting interventions. Results from communities like this that have undergone several rounds of MDA demonstrate the current inadequacy of solely using MDA for schistosomiasis control and elimination in persistent transmission hotspots.

During our study, lake water contact for as little as 5 minutes per day was significantly associated with the risk for S. mansoni infection, suggesting a high force of transmission in this community. Although the duration of water contact was significant, the frequency of water contact was not. People who never contact lake water have already been controlled for in the significant zero-minute duration of water contact group; therefore, anyone who contacts lake water, even for a few minutes, is at risk. It does not matter how often they have contact with water because they have likely already been exposed and have become infected. By living in a community that almost solely depends on lake water for their water source, the high frequency of lake water contact does not appear to substantially increase the risk of being infected because any contact could have resulted in infection. This heavy dependence is characteristic of many landing sites on large water bodies in sub-Saharan Africa that lack clean water for basic activities. ${ }^{31,32}$ Several activities like gathering water, ${ }^{33}$ swimming, fishing, fish mongering, and transit to different islands expose residents to lake water contact. $^{34}$ The majority of these activities are routine for most residents and usually involve approximately 5 minutes or more of water submersion. Therefore, because contact with the lake water is associated with infection, simple measures that enable people to collect water without entering it (such as using jetties) or telling people to wash their clothes while standing on the shore rather than in the water could be effective. Certain occupations require water access; therefore, additional interventions might be required, such as the use of wellies for farmers and boat jetties and dry landing sites for fishermen. With an increased cercariainfested lake water contact, we expect that the intensities of infection will increase; however, the size of the study population did not allow us to test this hypothesis robustly.

During this study, the majority of lake water contact involved gathering water. Other water sources such as boreholes are available in these communities. However, there is only one in Bugoto A and one in Bugoto B; they both frequently break, have long queues during the dry season, and are only used for safe drinking water, if at all. A community piped water system broke several years ago, but even when it was working, water was at a cost that was prohibitive to most community members. Therefore, lake water contact will remain unavoidable until a sustainable, popular, and affordable alternative is available. People need water to live, and the lake water is accessible and free, even if it is unsafe. There is an urgent need to make taps, boreholes, and/or wells more affordable, accessible, and safe so that people can be persuaded to use them (e.g., well water often tastes or lathers differently than lake water and can be less popu$\left.\operatorname{lar}^{35}\right)$. Education could help to ensure that people know that water that does not lather still washes the body and clothes well; however, personal taste preferences would likely be more difficult to change. ${ }^{36}$

We found that longer residence time in the community significantly increased the risk of acquiring S. mansoni infections. The intensity and morbidity associated with schistosomiasis are attributable to several exposures, with new additive infections acquired over time. ${ }^{4}$ The importance of residence time has been reported by others to be strongly associated with schistosomiasis infection. ${ }^{4,37}$ Targeting residents who have stayed in such a high-transmission hotspot community for more than $10 \%$ of their lifetime with MDA should help address individual morbidity and reduce schistosomiasis transmission. This could prove to be costeffective in terms of successfully targeting infected individuals and potential transmitters during the process of elimination.

An informed individual is expected to perform measures that would limit the risk of $S$. mansoni infection. We report that knowledge of schistosomiasis was actually positively associated with infection. This is the opposite of what we would expect. However, greater knowledge with reduced infections could only be expected if people have the option to change their behavior. It may be that education and community sensitization are working and are reaching people at high risk, thus increasing their knowledge about the disease, transmission, and symptoms; however, the absence of alternative options, ${ }^{38}$ such as safe water sources, limits their abilities to protect themselves because they still need to use lake water.

The reported low MDA compliance/use among community members may be attributed to the fatigue of the volunteer drug distributors, as reported by Knopp et al., ${ }^{39}$ not being offered treatment, ${ }^{25}$ individuals believing that they are not infected, ${ }^{25}$ conflicting agricultural activities, ${ }^{40}$ and the bitter taste of praziquantel. ${ }^{40} \mathrm{~A}$ positive association of previous MDA compliance with infection could be explained in the same way as we attributed the effects of knowledge, with those who are exposed being the ones who seek treatment and fear the consequences of the disease but have limited options to change their behavior after treatment and, therefore, are rapidly reinfected. The absence of MDA treatment and knowledge from the best-fit model could be attributable to the inability to change behaviors with treatment and knowledge; therefore, the actual exposure risk factors become the more important factors that drive the infection status. Alternatively, targeting treatments to SAC who are assumed to be at high risk and achieving high treatment coverage in schools and to PSAC who are not treated may mask the effects in the multivariant model. One limitation of this study was that the source of data used to ascertain praziquantel MDA compliance was interview answers, which are prone to recall bias. This recall bias, however, was minimized by showing participants tablets and describing the bitter taste. Furthermore, the total proportion of people who reported using praziquantel was similar to that reported by another study performed in the same village in $2017 .{ }^{25}$

A high proportion of community members (22.6\%) reported not always using latrines at work and $3.2 \%$ reported that they did not always use latrines at home. This could be attributed to a general lack of physical latrines or access to them at work, especially for fishermen and farmers, individuals being too far from home when a latrine was needed, or individuals not wanting to use latrines. The lack of a latrine door is not uncommon; therefore, latrines lack privacy. Additionally, latrines often lack cleanliness, particularly communal latrines that are common on landing sites, making them uninviting. Furthermore, when latrines are 
locked, they are impossible to use. All of these contribute to lower usage even when they are present. This inconsistency in latrine use behavior often leads to open defecation that contaminates the environment with feces laden with Schistosoma ova. ${ }^{41}$ Therefore, with few alternatives to lake water contact, contracting S. mansoni is likely to occur in such communities. ${ }^{42,43}$

A critical observation for program managers and policymakers is the high burden of potent $S$. mansoni eggs $(39.2 \%)$ in children younger than 5 years (PSAC). Our findings support other studies performed in the same community that reported a high disease burden for children younger than 5 years $^{44}$ and other studies across subSaharan Africa. ${ }^{45-47}$ Several scenarios explain the infection status of PSAC. PSAC contract the disease both actively and passively. Approximately half of the PSAC were reported to have visited the lake during the survey compared to $95 \%$ of SAC or approximately $90 \%$ of adults; however, this might be an underestimation and is based only on the answers provided by specific respondents. PSAC become actively infected by visiting the lake with their mothers/ guardians or older siblings to collect water for domestic use, bathing, washing clothes by the lake, and swimming. ${ }^{48}$ PSAC are not always with the main parent/guardian/survey respondent, and the siblings may take the PSAC to the lake without mentioning it to the specific survey respondents. Additionally, there were PSAC infected with S. mansoni whose parents/guardians reported that they did not visit the lake at all, indicating that the PSAC went without them, the lake visit information was not fully or accurately recorded, or the infection was passive. PSAC may passively contact cercaria-infested water when they are bathed in water collected from the lake with viable cercariae. One potential way to reduce this type of infection is to improve education among parents and telling them to avoid using lake water for 24 to 48 hours after collection so that the viable cercariae die before use or to use other immediate treatment options such as chlorination or filtration to kill the viable cercariae.

Most MDA has focused on SAC because of their vulnerability and the ease of access for treatments. However, in hotspot communities, young adults, in particular, have low praziquantel MDA compliance rates, ${ }^{25,49}$ high disease rates, practice open defecation, and could have an important role in the rapid infection and reinfection rates of SAC and PSAC. ${ }^{33}$ Although other studies have also observed high burdens in all ages, ${ }^{41,50}$ PSAC are not targeted by the control programs, and there is no approved pediatric formulation for this group. This maintains the status quo of health inequity, with PSAC heavily infected and showing morbidity ${ }^{51-53}$ before they even reach school age. Whatever the cause of these infections in PSAC, it is vital that they are treated. Based on the WHO grade levels of schistosomiasis and praziquantel MDA, this group (PSAC) deserves MDA.

\section{CONCLUSION}

S. mansoni is a public health problem in Bugoto community despite more than 14 years of MDA. Improving MDA coverage with targeted treatment strategies to at-risk residents who have lived more than $10 \%$ of their lifetime in the community would help control schistosomiasis and, therefore, help communities move toward the elimination of
S. mansoni as a public health problem. Because much of the population visits the lake for water collection, the provision of alternative water sources, such as boreholes and piped water, that are easily accessible and affordable would limit lake water contact and the associated risk. Building lake barriers such as jetties so that people can collect water without water contact, building platforms for washing clothes in the absence of alternative water sources, encouraging wellie use by farmers, encouraging the use of boats to reach dry land, and improving education methods surrounding delayed water contact after collection for PSAC bathing are suggested.

Received April 7, 2021. Accepted for publication August 6, 2021.

Published online October 18, 2021.

Note: Supplemental information appears at www.ajtmh.org.

Acknowledgments: We thank the community leaders and the District Vector Control Officer, Juma Nabonge, for their efforts in mobilizing the communities for this study. We thank the Village Health Teams for their assistance with data collection.

Financial support: Fieldwork was funded by the European Research Council Starting Grant (680088 SCHISTO_PERSIST to PHLL). PHLL and EMT are also supported by a Medical Research Council Global Challenges Research Fund (MRC GCRF) Foundation award (MR/ P025447/1). PHLL is also supported by Engineering and Physical Sciences Research Council Global Challenges Research Fund (EPSRC GCRF) awards (EP/T003618/1 and EP/R01437X/1).

Authors' addresses: Arinaitwe Moses, Moses Adriko, and Edridah M. Tukahebwa, Vector Control Division, Ministry of Health, Kampala, Uganda, E-mails: moses0772359814@gmail.com, adrikomoses@ gmail.com, and edmuheki@gmail.com. Brian Kibwika, Cavendish University Uganda, Kampala, Uganda, E-mail: bkibwika@cavendish. ac.ug. Christina L. Faust, Institute for Biodiversity, Animal Health and Comparative Medicine and Wellcome Centre for Integrative Parasitology, University of Glasgow, Glasgow, UK, E-mail: christina. faust@glasgow.ac.uk. Poppy H. L. Lamberton, Institute for Biodiversity, Animal Health and Comparative Medicine and Wellcome Centre for Integrative Parasitology, University of Glasgow, Glasgow, UK, E-mail: poppy.lamberton@glasgow.ac.uk.

This is an open-access article distributed under the terms of the Creative Commons Attribution (CC-BY) License, which permits unrestricted use, distribution, and reproduction in any medium, provided the original author and source are credited.

\section{REFERENCES}

1. Global Burden of Disease Study C, 2015. Global, regional, and national incidence, prevalence, and years lived with disability for 301 acute and chronic diseases and injuries in 188 countries, 1990-2013: a systematic analysis for the Global Burden of Disease Study 2013. Lancet 386: 743-800.

2. WHO, 2012. Accelerating Work to Overcome the Global Impact of Neglected Tropical Diseases. A Roadmap for Implementation. Geneva, Switzerland: World Health Organization.

3. Colley DG, Bustinduy AL, Secor WE, King CH, 2014. Human schistosomiasis. Lancet 383: 2253-2264.

4. Tukahebwa EM, Magnussen $P$, Madsen $H$, Kabatereine NB, Nuwaha F, Wilson S, Vennervald BJ, 2013. A very high infection intensity of Schistosoma mansoni in a Ugandan Lake Victoria fishing community is required for association with highly prevalent organ related morbidity. PLoS Negl Trop Dis 7: e2268.

5. Chami GF, Fenwick A, Bulte E, Kontoleon AA, Kabatereine NB, Tukahebwa EM, Dunne DW, 2015. Influence of Schistosoma mansoni and hookworm infection intensities on anaemia in Ugandan villages. PLoS Negl Trop Dis 9: e0004193.

6. Crellen $\mathrm{T}$, Walker M, Lamberton PH, Kabatereine NB, Tukahebwa EM, Cotton JA, Webster JP, 2016. Reduced efficacy 
of praziquantel against Schistosoma mansoni is associated with multiple rounds of mass drug administration. Clin Infect Dis 63: 1151-1159.

7. Faust $C$ et al., 2019. Two-year longitudinal survey reveals high genetic diversity of Schistosoma mansoni with adult worms surviving praziquantel treatment at the start of mass drug administration in Uganda. Parasit Vectors 12: 607.

8. Adriko M, Tinkitina B, Tukahebw EM, Standley CJ, Stothard JR, Kabatereine NB, 2018. The epidemiology of schistosomiasis in Lango region Uganda 60 years after Schwetz 1951: can schistosomiasis be eliminated through mass drug administration without other supportive control measures? Acta Trop 185: 412-418.

9. Walker JW, Kittur N, Binder S, Castleman JD, Drake JM, Campbell Jr CH, King CH, Colley DG, 2020. Environmental predictors of schistosomiasis persistent hotspots following mass treatment with praziquantel. Am J Trop Med Hyg 102: 328-338.

10. Clennon JA, King CH, Muchiri EM, Kariuki HC, Ouma JH, Mungai $P$, Kitron U, 2004. Spatial patterns of urinary schistosomiasis infection in a highly endemic area of coastal Kenya. Am J Trop Med Hyg 70: 443-448.

11. Wood CL et al., 2019. Precision mapping of snail habitat provides a powerful indicator of human schistosomiasis transmission. Proc Natl Acad Sci USA 116: 23182.

12. Kabuyaya M, Chimbari MJ, Manyangadze T, Mukaratirwa S, 2017. Schistosomiasis risk factors based on the infection status among school-going children in the Ndumo area, Mkhanyakude district, South Africa. S Afr J Infect Dis 32: 67-72.

13. Angora EK, Boissier J, Menan H, Rey O, Tuo K, Touré AO, Coulibaly JT, Méité A, Raso G, N'Goran EK, 2019. Prevalence and risk factors for schistosomiasis among schoolchildren in two settings of Côte d'Ivoire. Trop Med Int Health 4: 110.

14. Grimes JE, Croll D, Harrison WE, Utzinger J, Freeman MC, Templeton MR, 2015. The roles of water, sanitation and hygiene in reducing schistosomiasis: a review. Parasit Vectors 8: 156.

15. Assefa A, Dejenie T, Tomass Z, 2013. Infection prevalence of Schistosoma mansoni and associated risk factors among schoolchildren in suburbs of Mekelle city, Tigray, northern Ethiopia. Ethiop J Health Sci 5: 174-188.

16. Muhumuza S, Kitimbo G, Oryema-Lalobo M, Nuwaha F, 2009. Association between socioeconomic status and schistosomiasis infection in Jinja District, Uganda. Trop Med Int Health 14: 612-619.

17. Ministry of Health, 2017. Uganda Master Plan for National Neglected Tropical Diseases Programmes: 2017-2022. Kampala, Uganda: Republic of Uganda Ministry of Health.

18. World Health Organization, 2001. Schistosomiasis and Soiltransmitted Helminth Infections WHA54.19. Geneva, Switzerland: WHO.

19. Kabatereine NB, Brooker S, Koukounari A, Kazibwe F, Tukahebwa EM, Fleming FM, Zhang Y, Webster JP, Stothard JR, Fenwick A, 2007. Impact of a national helminth control programme on infection and morbidity in Ugandan schoolchildren. Bull World Health Organ 85: 91-99.

20. Deol A et al., 2019. Schistosomiasis - assessing progress towards the 2020 and 2025 goals. N Engl J Med 381: 25192528.

21. Trienekens SC, Faust CL, Meginnis K, Pickering L, Ericsson O, Nankasi A, Moses A, Tukahebwa EM, Lamberton PH, 2020. Impacts of host gender on Schistosoma mansoni risk in rural Uganda - a mixed-methods approach. PLOS Negl Trop Dis 14: e0008266.

22. Kittur N, Binder S, Campbell Jr CH, King CH, Kinung'hi S, Olsen A, Magnussen P, Colley DG, 2017. Defining persistent hotspots: areas that fail to decrease meaningfully in prevalence after multiple years of mass drug administration with praziquantel for control of schistosomiasis. Am J Trop Med Hyg 97: 1810-1817.

23. WHO, 2020. Ending the Neglect to Attain the Sustainable Development Goals: A Road Map for Neglected Tropical Diseases 2021-2030. Geneva, Switzerland: World Health Organization.

24. Perez-Saez J et al., 2015. A theoretical analysis of the geography of schistosomiasis in Burkina Faso highlights the roles of human mobility and water resources development in disease transmission. PLoS Negl Trop Dis 9: e0004127.

25. Adriko M, Faust C, Carruthers L, Moses A, Tukahebwa E, Lamberton P, 2018. Low praziquantel treatment coverage for Schistosoma mansoni in Mayuge District, Uganda, Due to the absence of treatment opportunities, rather than systematic non-compliance. Trop Med Infect Dis 3: 111.

26. Uganda Bureau of Statistics, 2016. The National Population and Housing Census 2014 - Main Report, Kampala, Uganda. Available at: https://www.ubos.org/wp-content/uploads/ publications/03_20182014_National_Census_Main_Report. pdf.

27. Lamberton PH, Kabatereine NB, Oguttu DW, Fenwick A, Webster JP, 2014. Sensitivity and specificity of multiple Kato-Katz thick smears and a circulating cathodic antigen test for Schistosoma mansoni diagnosis pre- and post-repeated-praziquantel treatment. PLoS Negl Trop Dis 8: e3139.

28. Kish L, 1995. Survey Sampling. Wiley-Interscience, 664 pp.

29. Uganda Bureau of Statistics (UBOS) and ICF International Inc., 2012. Uganda Demographic and Health Survey 2011. Kampala, Uganda: UBOS and Calverton, MD: ICF International Inc. Available at: http://www.measuredhs.com.

30. Filmer D, Pritchett LH, 2001. Estimating wealth effects without expenditure data-or tears: an application to educational enrollments in states of India. Demography 38: 115-132.

31. World Health Organization, 2015. Assessing the Epidemiology of Soil-transmitted Helminths during a Transmission Assessment Survey in the Global Programme for the Elimination of the Lymphtic Filariasis. Available at: http://apps.who.int/iris/bitstream/10665/153240/1/9789241508384_eng.pdf. Accessed August 4, 2015.

32. World Health Organization, 2015. Investing to Overcome the Global Impact of Neglected Tropical Diseases: Third Who Report on neglected Tropical Diseases. Geneva, Switzerland: WHO.

33. Kulinkina AV, Walz Y, Koch M, Biritwum N-K, Utzinger J, Naumova EN, 2018. Improving spatial prediction of Schistosoma haematobium prevalence in southern Ghana through new remote sensors and local water access profiles. PLOS Negl Trop Dis 12: e0006517.

34. Standley CJ, Kabatereine NB, Lange CN, Lwambo NJ, Stothard $\mathrm{JR}$, 2010. Molecular epidemiology and phylogeography of Schistosoma mansoni around Lake Victoria. Parasitology 137: 1937-1949.

35. Opryszko MC, 2012. The Household Effect: An Independent Longitudinal Evaluation of Water-Vending Kiosks in Rural Ghana. Baltimore, MD: The Johns Hopkins University.

36. Kosinski KC, Kulinkina AV, Abrah AFA, Adjei MN, Breen KM, Chaudhry HM, Nevin PE, Warner SH, Tendulkar SA, 2016. A mixed-methods approach to understanding water use and water infrastructure in a schistosomiasis-endemic community: case study of Asamama, Ghana. BMC Public Health 16: 322.

37. Assefa LM, Crellen T, Kepha S, Kihara JH, Njenga SM, Pullan RL, Brooker SJ, 2014. Diagnostic accuracy and costeffectiveness of alternative methods for detection of soiltransmitted helminths in a post-treatment setting in western Kenya. PLoS Negl Trop Dis 8: e2843.

38. Kloos H, Fulford AJC, Butterworth AE, Sturrock RF, Ouma JH, Kariuki HC, Thiongo FW, Dalton PR, Klumpp RK, 1997. Spatial patterns of human water contact and Schistosoma mansoni transmission and infection in four rural areas in Machakos District, Kenya. Soc Sci Med 44: 949-968.

39. Knopp S et al., 2016. Praziquantel coverage in schools and communities targeted for the elimination of urogenital schistosomiasis in Zanzibar: a cross-sectional survey. Parasit Vectors 9: 5.

40. Coulibaly J, Ouattara M, Barda B, Utzinger J, N'Goran E, Keiser $\mathrm{J}, 2018$. A rapid appraisal of factors influencing praziquantel treatment compliance in two communities endemic for schistosomiasis in Côte d'Ivoire. Trop Med Int Health 3: 69.

41. Exum NG, Kibira SP, Ssenyonga R, Nobili J, Shannon AK, Ssempebwa JC, Tukahebwa EM, Radloff S, Schwab KJ, Makumbi FE, 2019. The prevalence of schistosomiasis in Uganda: a nationally representative population estimate to 
inform control programs and water and sanitation interventions. PLoS Negl Trop Dis 13: e0007617.

42. Munisi DZ, Buza J, Mpolya EA, Angelo T, Kinung'hi SM, 2017. Knowledge, attitude, and practices on intestinal schistosomiasis among primary schoolchildren in the Lake Victoria basin, Rorya District, north-western Tanzania. BMC Public Health 17: 731.

43. Traore I, 2013. Geographic determinants of human schistosomiasis transmission in the Sourou Valley, Burkina Faso. PhD Dissertation, der Georg-August-Universität Göttingen.

44. Betson M, Sousa-Figueiredo JC, Rowell C, Kabatereine NB, Stothard JR, 2010. Intestinal schistosomiasis in mothers and young children in Uganda: investigation of field-applicable markers of bowel morbidity. Am J Trop Med Hyg 83: 1048-1055.

45. Bustinduy AL, Friedman JF, Kjetland EF, Ezeamama AE, Kabatereine NB, Stothard JR, King CH, 2016. Expanding praziquantel (PZQ) access beyond mass drug administration programs: paving a way forward for a pediatric PZQ formulation for schistosomiasis. PLoS Negl Trop Dis 10: e0004946.

46. Sousa-Figueiredo JC, Pleasant J, Day M, Betson M, Rollinson D, Montresor A, Kazibwe F, Kabatereine NB, Stothard JR, 2010. Treatment of intestinal schistosomiasis in Ugandan preschool children: best diagnosis, treatment efficacy and side-effects, and an extended praziquantel dosing pole. Int Health 2: 103-113.

47. Stothard JR, Sousa-Figueiredo JC, Betson M, Bustinduy A, Reinhard-Rupp J, 2013. Schistosomiasis in African infants and preschool children: let them now be treated! Trends Parasitol 29: 197-205.

48. Ekpo UF, Laja-Deile A, Oluwole AS, Sam-Wobo SO, Mafiana CF, 2010. Urinary schistosomiasis among preschool children in a rural community near Abeokuta, Nigeria. Parasit Vectors 3: 58.

49. Korir H, Riner D, Kavere E, Omondi A, Landry J, Kittur N, Ndombi E, Ondigo B, Secor W, Karanja D, 2018. Young adults in endemic areas: an untreated group in need of school-based preventive chemotherapy for schistosomiasis control and elimination. Trop Med Infect Dis 3: 100.

50. Nalugwa A, Olsen A, Tukahebwa M, Nuwaha F, 2015. Intestinal schistosomiasis among preschool children along the shores of Lake Victoria in Uganda. Acta Trop 142: 115-121.

51. Davis SM, Wiegand RE, Mulama F, Kareko El, Harris R, Ochola E, Samuels AM, Rawago F, Mwinzi PM, Fox LM, 2015. Morbidity associated with schistosomiasis before and after treatment in young children in Rusinga Island, western Kenya. Am J Trop Med Hyg 92: 952-958.

52. Sousa-Figueiredo JC, Betson M, Atuhaire A, Arinaitwe M, Navaratnam AM, Kabatereine NB, Bickle Q, Stothard JR, 2012. Performance and safety of praziquantel for treatment of intestinal schistosomiasis in infants and preschool children. PLOS Negl Trop Dis 6: e1864.

53. Stothard JR, Sousa-Figueiredo JC, Betson M, Adriko M, Arinaitwe M, Rowell C, Besiyge F, Kabatereine NB, 2011. Schistosoma mansoni infections in young children: when are schistosome antigens in urine, eggs in stool and antibodies to eggs first detectable? PLoS Negl Trop Dis 5: e938. 